\title{
Coherent spin rotation-induced zero thermal expansion in MnCoSi-based spiral magnets
}

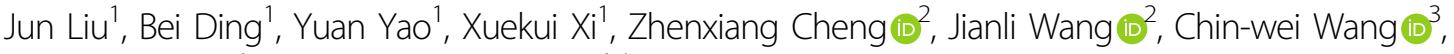 \\ Guangheng $\mathrm{Wu}^{1}$ and Wenhong Wang $\mathbb{D}^{1,4}$
}

\begin{abstract}
Materials exhibiting zero thermal expansion (ZTE), namely, volume invariance with temperature change, can resist thermal shock and are highly desired in modern industries for high-precision components. However, pure ZTE materials are rare, especially those that are metallic. Here, we report the discovery of a pure metallic ZTE material: an orthorhombic $\mathrm{Mn}_{1-x} \mathrm{Ni}_{x} \mathrm{CoSi}$ spiral magnet. The introduction of $\mathrm{Ni}$ can efficiently enhance the ferromagnetic exchange interaction and construct the transition from a spiral magnetic state to a ferromagnetic-like state in MnCoSi-based alloys. Systematic in situ neutron powder diffraction revealed a new cycloidal spiral magnetic structure in the bc plane in the ground state, which transformed to a helical spiral in the $a b$ plane with increasing temperature. Combined with Lorentz transmission electron microscopy techniques, the cycloidal and helical spin order coherently rotated at varying periods along the $c$-axis during the magnetic transition. This spin rotation drove the continuous movement of the coupled crystalline lattice and induced a large negative thermal expansion along the $a$-axis, eventually leading to a wide-temperature ZTE effect. Our work not only introduces a new ZTE alloy but also presents a new mechanism by which to discover or design ZTE magnets.
\end{abstract}

\section{Introduction}

It is well known that the inherent anharmonicity of phonon vibrations triggers the volume expansion of most solids upon heating. However, when the crystalline lattice couples with ferroelectricity, magnetism, and charge transfer, anomalous behavior during large temperature fluctuations, namely, negative thermal expansion (NTE) behavior, may be realized ${ }^{1-4}$. Combining NTE materials with positive thermal expansion (PTE) materials can reduce the overall coefficient of thermal expansion (CTE) and lead to overall zero thermal expansion (ZTE) composites, which are of great importance in industrial applications as structural components, electronic devices, and high-precision instruments ${ }^{1,5-7}$. Unfortunately, such

\footnotetext{
Correspondence: Wenhong Wang (Wenhong.Wang@iphy.ac.cn)

${ }^{1}$ Beijing National Laboratory for Condensed Matter Physics, Institute of Physics,

Chinese Academy of Sciences, 100190 Beijing, China

${ }^{2}$ Institute for Superconducting and Electronic Materials, Innovation Campus,

University of Wollongong, Squires Way, North Wollongong, NSW 2500,

Australia

Full list of author information is available at the end of the article
}

ZTE composite materials have high internal stresses that can cause microcracking during thermal cycling, which significantly diminishes their mechanical performance and lifetime. This problem can be overcome with singlephase ZTE materials with homogeneous internal structures, especially in metallic form. In addition to Invar alloys $^{8}$, a small number of such single-phase ZTE alloys and compounds have been discovered thus far, such as $\mathrm{Mn}_{1-x} \mathrm{Co}_{x} \mathrm{~B}^{9}, \quad \mathrm{YbGaGe}^{10}, \quad \mathrm{LaFe}_{10.6} \mathrm{Si}_{2.4}{ }^{11}, \quad \mathrm{Ho}_{2} \mathrm{Fe}_{16} \mathrm{Cr}^{12}$, $\mathrm{ErFe}_{10} \mathrm{~V}_{1.6} \mathrm{Mo}_{0.4}{ }^{13}$, and $\mathrm{RFe}_{2}$-based compounds ${ }^{14-16}$.

The most common of these materials are magnetic alloys, for which the ZTE behavior is intimately associated with spontaneous magnetic ordering, known as the magnetovolume effect (MVE). The MVE originating from spin-lattice coupling may weaken or even compensate for anharmonic lattice variations and lead to abnormal thermal expansion behavior near the magnetic ordering temperature ${ }^{17,18}$. Here, we present a new class of ZTE materials, $\mathrm{MnCoSi}$-based metamagnetic alloys, consisting of a homogeneous phase. The ternary equiatomic $\mathrm{MnCoSi}$

\section{(c) The Author(s) 2021}

(c) (i) Open Access This article is licensed under a Creative Commons Attribution 4.0 International License, which permits use, sharing, adaptation, distribution and reproduction cc) in any medium or format, as long as you give appropriate credit to the original author(s) and the source, provide a link to the Creative Commons license, and indicate if changes were made. The images or other third party material in this article are included in the article's Creative Commons license, unless indicated otherwise in a credit line to the material. If material is not included in the article's Creative Commons license and your intended use is not permitted by statutory regulation or exceeds the permitted use, you will need to obtain permission directly from the copyright holder. To view a copy of this license, visit http://creativecommons.org/licenses/by/4.0/. 


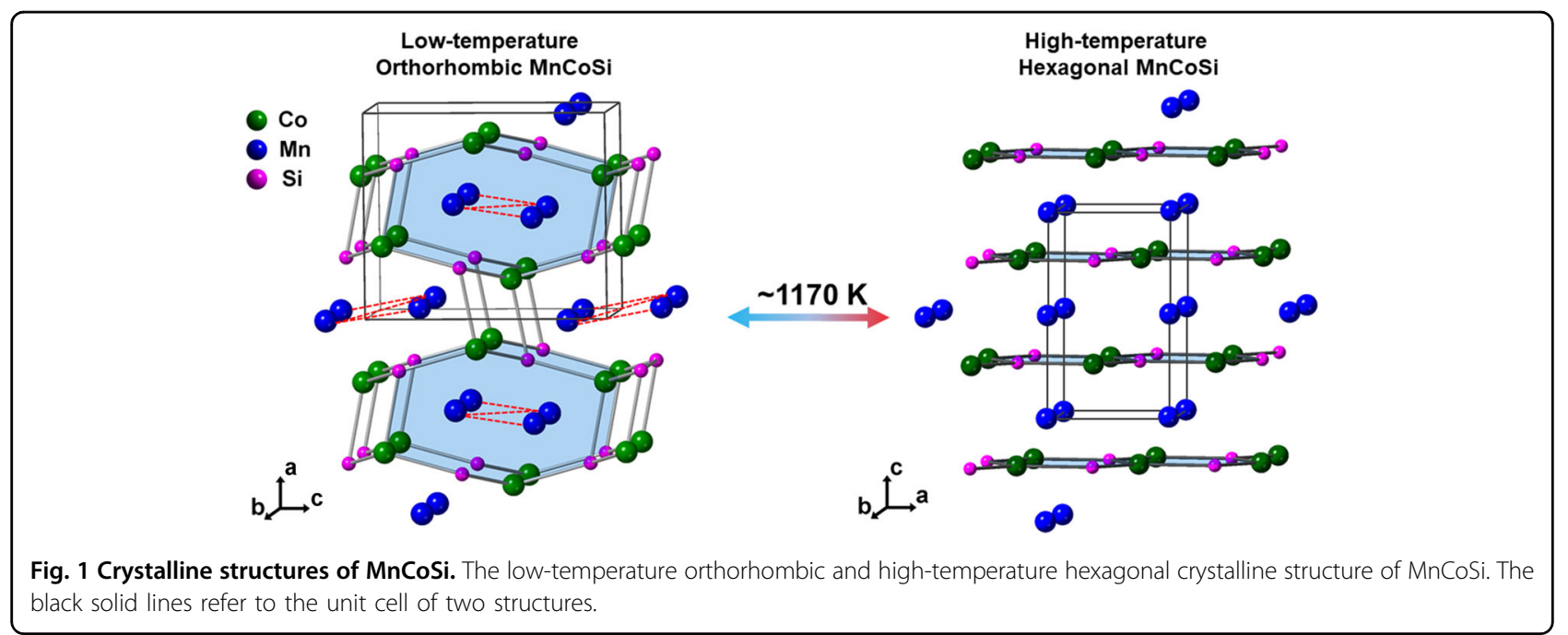

alloy crystallizes with an orthorhombic structure from a honeycomb hexagonal structure after experiencing a martensitic transition at high temperature (Fig. 1 and Supplementary Fig. S1). The shortest Co-Si bonds yield wrinkled eight-membered rings, in which $\mathrm{Mn}-\mathrm{Mn}$ zig-zag chains are embedded. The interconnected $\mathrm{Co}-\mathrm{Si}$ contacts along the [100] direction form the basic rigid skeleton. Due to the critical nearest $\mathrm{Mn}-\mathrm{Mn}$ separation, $\mathrm{MnCoSi}$ alloys possess a ground state of nonlinear antiferromagnetism (AFM) and a magnetic-field-induced magnetoelastic transition ${ }^{19,20}$, during which a large inverse magnetocaloric effect and giant magnetostrictive effect are realized ${ }^{21-23}$. In this study, we find that the helical magnetic structure of $\mathrm{MnCoSi}$, as is widely recognized, transforms to cycloidal spiral AFM at low temperature. Moreover, we report that when the $\mathrm{MnCoSi}$ system is tuned by minimal $\mathrm{Ni}$ introduction, an ultralow CTE over a wide temperature range can be achieved. The combination of X-ray diffraction (XRD), neutron powder diffraction (NPD), and Lorentz transmission electron microscopy (TEM) techniques reveal a new mechanism underlying ZTE: coherent spin rotation of the spiral magnetic structures.

\section{Materials and methods \\ Sample synthesis}

Polycrystalline $\mathrm{Mn}_{1-x} \mathrm{Ni}_{x} \mathrm{CoSi}(x=0,0.010,0.015$, $0.017,0.020$, and 0.025$)$ samples were prepared by arc melting the appropriate amounts of high-purity raw materials under a purified argon atmosphere three times. Then, the as-cast samples were sealed in evacuated quartz ampoules and annealed at $1123 \mathrm{~K}$ for $60 \mathrm{~h}$ before being slowly cooled to room temperature over $72 \mathrm{~h}$. The slow-cooling treatment guaranteed magnetic homogeneity ${ }^{24}$.

\section{Magnetization, XRD, and NPD characterization}

The magnetic properties were characterized by a superconducting quantum interference device (SQUID, Quantum Design MPMS XL7) with the reciprocating sample option. The temperature dependence of the powder XRD (Rigaku, Smartlab) was collected using a low-temperature chamber. For each measurement at a specified temperature, the powder sample was maintained for $20 \mathrm{~min}$ to reach heat equilibrium. In situ variabletemperature NPD measurements $(\lambda=1.622 \AA)$ in the heating process were carried out on the Wombat beamline at the OPAL facility of the Australian Nuclear Science and Technology Organization (ANSTO). Structural refinements of the XRD and NPD patterns were performed using the Rietveld refinement method, and the irreducible representation analysis of the magnetic structure was carried out using the BASIREPS program, both implemented in the FULLPROF package ${ }^{25,26}$.

\section{Lorentz TEM measurements}

The thin plates for Lorentz TEM observations were prepared with a focused ion beam. The temperature dependence of the magnetic domain structures was observed in a JEOL-dedicated Lorentz TEM (JEOL2100F) equipped with liquid-nitrogen holders. To determine the in-plane spin distribution of the magnetic texture, three sets of images with under, over, and just (or zero) focal lengths were recorded with a charge-coupled device (CCD) camera, and then the high-resolution in-plane magnetization distribution map was obtained using the commercial software QPt on the basis of the transport-ofintensity equation (TIE) equation. The orientation of the in-plane magnetization was depicted based on the color wheel. The crystalline orientation for the thin plate was determined by selected area electron diffraction (SAED). 


\section{Results}

According to the atomic occupancy rules in $\mathrm{MnCoSi}$ alloys $^{23}$, when $\mathrm{Ni}$ atoms nominally substitute for $\mathrm{Mn}$ atoms, $\mathrm{Ni}$ with more valence electrons preferably occupies the Co site, and then a partial Co atom occupies the $\mathrm{Mn}$ site. Thus, the occupation formula of the $\mathrm{Mn}_{1-x} \mathrm{Ni}_{x} \mathrm{CoSi}$ system should be written as $\left(\mathrm{Mn}_{1-x} \mathrm{Co}_{x}\right)\left(\mathrm{Co}_{1-x} \mathrm{Ni}_{x}\right) \mathrm{Si}$. The atomic occupation can also be confirmed by NPD refinement, as shown in Supplementary Fig. S2. Based on the atomic occupation, the lattice parameters and unitcell volume of the $\mathrm{Mn}_{1-x} \mathrm{Ni}_{x} \mathrm{CoSi}$ system are obtained from the refinement of XRD patterns, as shown in Fig. 2. For further details of the refinement, see Supplementary Fig. S3 and Supplementary Table S1. With decreasing temperature, the lattice parameters $b$ and $c$ typically decrease, while the lattice parameter $a$ shows an NTE effect. Moreover, with the introduction of $\mathrm{Ni}$, the expansion of the lattice parameter $a$ upon cooling shifts to a low temperature and changes dramatically. As a result, the effect of the shrinkage of $b$ and $c$ on the unit-cell volume is compensated for; thus, ZTE behavior is realized in Ni-containing samples, as presented in Fig. 2d. The reliability of anisotropic thermal expansion and ZTE can also be confirmed from the NPD results (Supplementary Fig. S4). Notably, stoichiometric MnCoSi exhibits a linear PTE in the studied temperature range with a slight inflection at $\sim 230 \mathrm{~K}$. When $\mathrm{Ni}$ substitutes for minimal
Mn, the samples, such as those with contents of $x=0.020$ and 0.025 , exhibit ultralow CTEs $\left(\alpha_{l}=6.9 \times 10^{-7}\right.$ and $1.3 \times 10^{-7} \mathrm{~K}^{-1}$, respectively) over a wide temperature range $(10-190 \mathrm{~K}$ and $10-170 \mathrm{~K}$, respectively) after experiencing a normal PTE and a weak NTE, respectively, that are approximately one-order smaller than that of the Invar alloy of $\mathrm{Fe}_{65} \mathrm{Ni}_{35}\left(\alpha_{l}=1.4 \times 10^{-6} \mathrm{~K}^{-1}\right)$. The calculated CTEs and corresponding working temperature ranges are listed in Supplementary Table S2 in the Supplementary Materials.

As mentioned before, the thermal expansion properties of magnetic materials can be affected by the MVE. The temperature dependence of the magnetization $(\mathrm{M}-\mathrm{T})$ curve during zero-field cooling (ZFC) and field cooling (FC) processes in Fig. 3a and Supplementary Fig. S5 shows that the weak magnetization of nonlinear AFM increases slowly with increasing temperature; this behavior is interrupted by the advent of paramagnetism (PM) in stoichiometric $\mathrm{MnCoSi}$ alloys. Hence, the long-range magnetic order cannot be maintained above $T_{0} \sim 387 \mathrm{~K}$, where $T_{0}$ represents the order-to-disorder transition temperature. It is widely reported that the introduction of external elements can effectively tune the magnetic state of TMnX ( $\mathrm{T}=$ transition metal, $\mathrm{X}=\mathrm{Si}$ or Ge) alloys ${ }^{19,27}$. In this work, the $\mathrm{M}-\mathrm{T}$ curves of the studied $\mathrm{Mn}_{1-x} \mathrm{Ni}_{x-}$ $\mathrm{CoSi}$ system indicate that minimal $\mathrm{Ni}$ addition strengthens the ferromagnetic (FM) interaction, leading to a rise
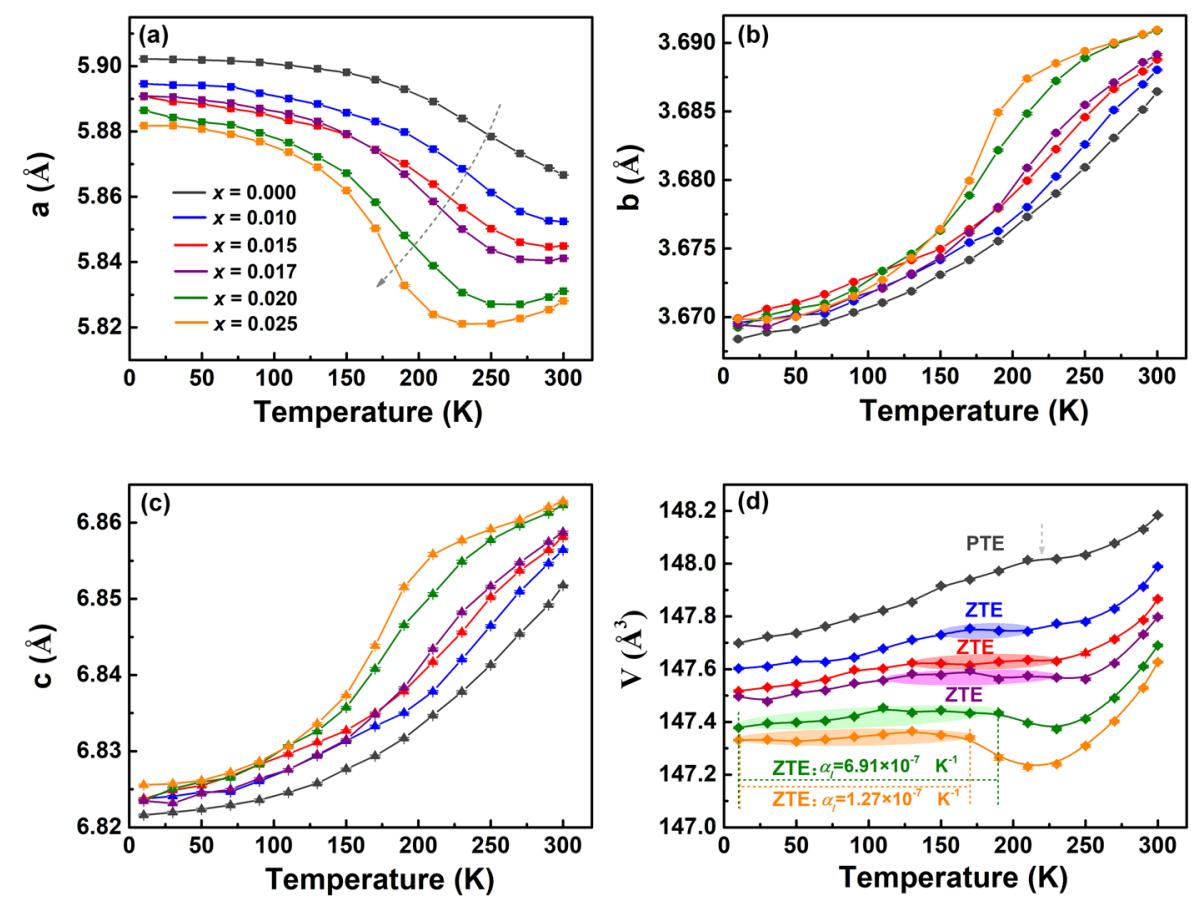

Fig. 2 Lattice parameters and unit-cell volume of the $\mathbf{M n}_{1-\boldsymbol{x}} \mathbf{N i}_{\boldsymbol{x}} \mathbf{C o S i}$ system. Temperature dependence of the lattice parameters (a) $a$, (b) $b$, and (c) $c$ and (d) unit-cell volume $V$ obtained from Rietveld refinement of the XRD patterns of the $\mathrm{Mn}_{1-x} \mathrm{Ni}_{x} \mathrm{CoSi}$ system. The linear CTEs of the Mn ${ }_{1-x} \mathrm{Ni} \mathrm{i}_{x} \mathrm{CoSi}$ system were calculated by $a_{l}=1 / 3 \cdot d V /\left(V_{0} \cdot d T\right)$. The ZTE working temperature range is indicated in $(\mathbf{d})$. 

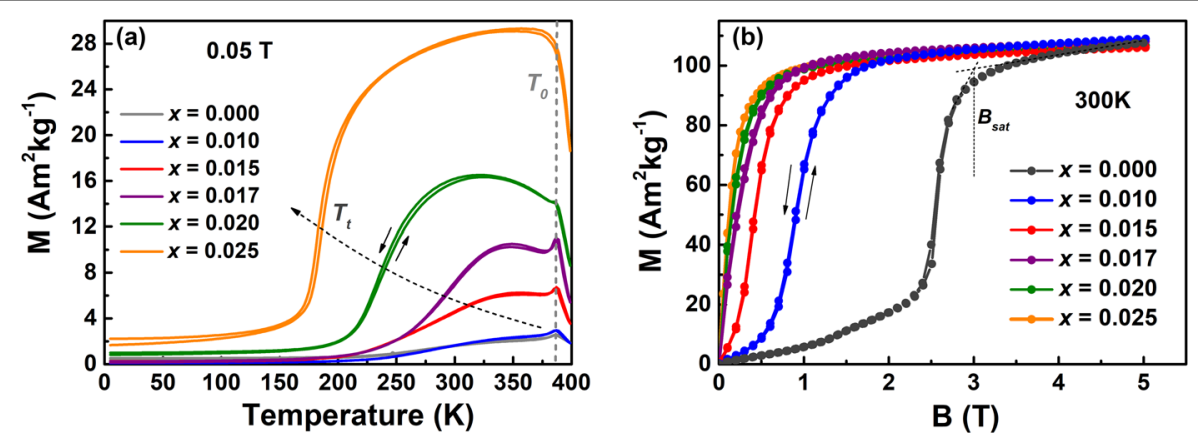

Fig. 3 Magnetic properties of $\mathbf{M n}_{1-x} \mathbf{N i}_{x}$ CoSi system. a M-T curves measured at $0.05 T$ during the ZFC and FC processes and $\mathbf{b}$ magnetization curves with increasing and decreasing magnetic fields at $300 \mathrm{~K}$ for the $\mathrm{Mn}_{1-x} \mathrm{Ni}_{x} \mathrm{CoSi}$ system.

in the hidden thermal-induced magnetic transition similar to that in Fe-substituted $\mathrm{MnCoSi}$ alloys 28 . For the $x=$ 0.020 sample, the AFM state smoothly transitions to the FM-like state, which is accompanied by a relatively large increase in magnetization. The magnetic transition temperature $T_{t}$, defined as the inflection point in the M-T curve, is presented in Supplementary Table S2 in the Supplementary Materials and gradually decreases with increasing $\mathrm{Ni}$ content. In addition, the establishment of FM coupling can be examined by the magnetization behavior. As shown by the room-temperature magnetization curves in Fig. 3b, the metastable nonlinear AFM can be easily destroyed by applying a magnetic field. A second-order and nonhysteretic metamagnetic transition displaying a sharp increase in magnetization is clearly seen in stoichiometric $\mathrm{MnCoSi}$ and tends to saturate at $B_{\text {sat }} \sim 3.0 \mathrm{~T}$. With increasing $\mathrm{Ni}$ content, $B_{\text {sat }}$ decreases (Supplementary Table S2), and the field-induced metamagnetism vanishes for the $x=0.020$ and 0.025 samples, where only FM-like behavior is exhibited. The macroscopic magnetic measurements indicate that the introduction of Ni can trigger a magnetic transition from the weak nonlinear AFM state to the FM-like state, where NTE or ZTE emerges.

To further determine the evolution of the magnetic structure, the temperature-dependent NPD was performed on the $\mathrm{Mn}_{1-x} \mathrm{Ni}_{x} \mathrm{CoSi}$ system. In the NPD patterns of the studied $x=0.000,0.015$, and 0.020 samples in Fig. 4 and Supplementary Fig. S6, with decreasing temperature, additional peaks at low diffraction angles corresponding to the magnetic satellite reflections gradually appear and then split or merge, manifesting the possible AFM order. Specifically, the refinement of isothermal NPD data at selected temperatures is also shown. At $450 \mathrm{~K}$, only the nuclear scattering of the orthorhombic space group is indexed because the sample is in a disordered paramagnetic state. When the sample enters the spin-ordered state below $T_{0}$, the magnetic reflections can be indexed by the nonlinear magnetic structure. As the temperature further decreases lower than $190 \mathrm{~K}$ for sample $x=0.020$, magnetic diffraction peaks of $(101)^{-},(-101)^{-},(202)^{-}$and $(-202)^{-}$at $\sim 2 \theta=18^{\circ}$ and $40^{\circ}$ gradually become prominent, which may be indicative of the change in the nonlinear magnetic structure.

Assisted by symmetry arguments ${ }^{29}$, the best-fit model indicates that ordered and equal moments are detected on Mn atoms $\left(\sim 3 \mu_{B}\right)$ or Co atoms $\left(\sim 0.6 \mu_{B}\right)$ (Supplementary Fig. S7) at $3 \mathrm{~K}$, for which the cycloidal spiral magnetic arrangement lying in the $b c$ plane achieves an incommensurate propagation vector $\boldsymbol{k}=\left(0,0, k_{c}\right)$ for the $x=$ 0.020 sample, as shown in Fig. $5 \mathrm{a}$, b. This spiral magnetic structure is different from the helical magnetic structure in the literature (two NPD refinements are presented and discussed in Supplementary Fig. S8) ${ }^{20}$, which was also reported by $\mathrm{O}$. Baumfeld before ${ }^{30}$. Specifically, although all the atoms occupy the same crystal site (Wyckoff position $4 c(x, 1 / 4, z))$, the wave vector group splits the magnetic $\mathrm{Mn}$ and Co positions into four magnetic spirals with identical $k$ values: $\langle\mathrm{Mn} 1, \mathrm{Mn} 3>,<\mathrm{Mn} 2, \mathrm{Mn} 4>,<\mathrm{Co} 1$, Co3 $>$, and $\langle\mathrm{Co} 2, \mathrm{Co} 4>$, and the magnetic spin in each cycloidal spiral rolls with a fixed angle along the $c$-axis. As the main carriers of the magnetic moment, the two magnetic spirals of $\mathrm{Mn}$ atoms exhibit obvious phase differences, indicating different spin orientations in the $x=$ 0.020 sample at the ground state. As the temperature increases, the four groups of cycloidal AFM transform to four groups of helical magnetic structures at approximately $190 \mathrm{~K}$ for the $x=0.020$ sample. As illustrated in Fig. $5 c, d$, the spin in each helix of the helical magnetic structure rotates in the $a b$ plane by a certain angle from layer to layer along the $c$-axis. Therefore, the envelope of the projection of magnetic moments in the $b c$ plane is sinusoidally modulated, as shown in Fig. $5 \mathrm{c}$. At $300 \mathrm{~K}$, the two $\mathrm{Mn}$ helices rotate almost synchronously and show a small phase difference in the $x=0.020$ sample.

Figure 6 shows the related angles between different magnetic spins from the analysis of NPD refinement. Based on their atomic positions, the nearest $\mathrm{Mn}$ or Co atoms in 


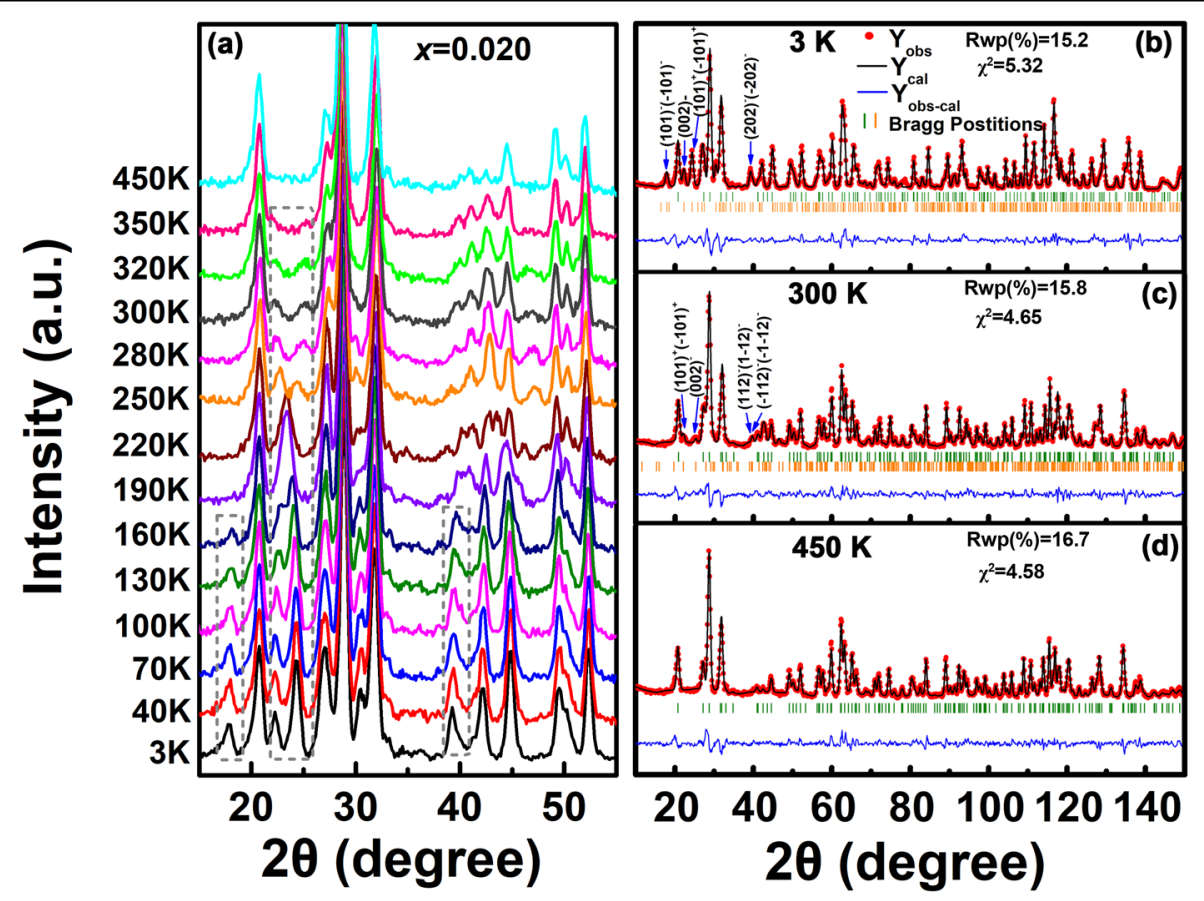

Fig. 4 NPD patterns and the refinement results. a Temperature dependence of the NPD patterns for the $x=0.020$ sample. The main magnetic diffraction peaks are indicated in the gray dashed box. Specific NPD patterns at b $3 \mathrm{~K}, \mathbf{c} 300 \mathrm{~K}$, and d $450 \mathrm{~K}$. Experimental (red) and calculated NPD patterns (black) and their difference profiles (blue) are shown. Vertical lines indicate the peak positions for the nuclear (green) and magnetic (orange) reflections of the MnCoSi phase.

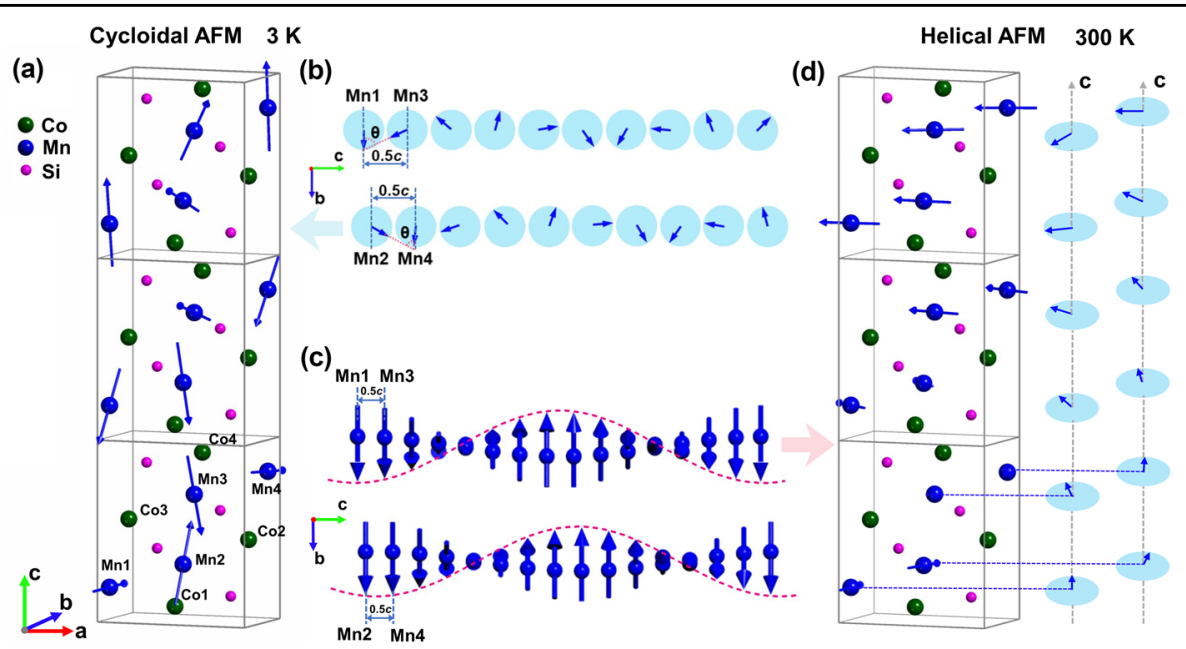

Fig. $\mathbf{5}$ Cycloidal and helical magnetic structures of the $\boldsymbol{x}=\mathbf{0 . 0 2 0}$ sample. a The cycloidal spiral magnetic structure of cell $a \times b \times 3 c$ at $3 \mathrm{~K}$. Two groups of $\mathrm{Mn}(\mathbf{b})$ cycloidal $(3 \mathrm{~K})$ and (c) helical ( $300 \mathrm{~K}$ ) magnetic structures viewed from the $a$-axis. For clarity, $\mathrm{Mn} 1$ and $\mathrm{Mn} 3$ (Mn2 and Mn4) atoms are moved and arranged along the $c$-axis. $\mathbf{d}$ The helical magnetic structure of cell $a \times b \times 3 c$ at $300 \mathrm{~K}$. The moment of the Co atom is smaller than the diameter of the atomic symbol and is invisible in the plots.

each helix have $z$-coordinates that differ by 0.5 , implying a phase difference of $k_{c} / 2$ between spins and an angle between the adjacent $\mathrm{Mn}$ or Co atoms of $180^{\circ} k_{c}$. With a temperature increase from 3 to $350 \mathrm{~K}$, the propagation vector component $k_{c}$ of the cycloidal or helical AFM structure for the studied samples decreases, with a relatively abrupt change near $T_{t}$ (Fig. 6a), indicating a decrease in the angle between the adjacent spins $\left(\theta_{\mathrm{Mn1}-\mathrm{Mn} 3} /\right.$ $\left.\theta_{\mathrm{Mn2}-\mathrm{Mn} 4}\right)$ in a cycloidal or helical magnetic chain and, correspondingly, an elongation of the magnetic spiral period. The temperature-dependent $\theta_{\mathrm{Mn} 1-\mathrm{Mn} 3}$ shows more obvious variation in $\mathrm{Ni}$-containing samples, and the angle 


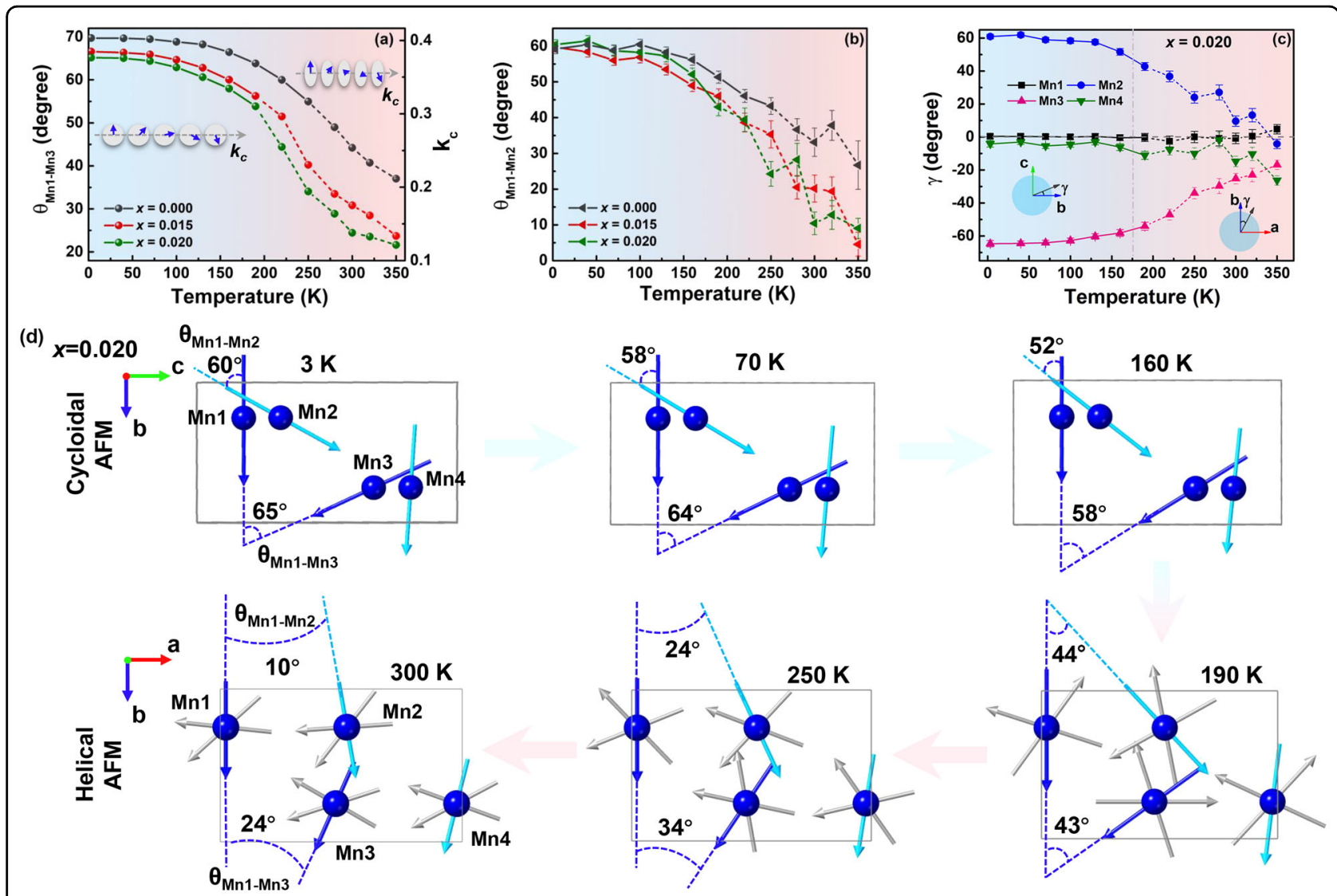

Fig. 6 Evolution of the angles between magnetic spins. Temperature dependence of the angles between a adjacent Mn1 and Mn3 magnetic spins $\left(\theta_{\mathrm{Mn1}-\mathrm{Mn3}}=180^{\circ} \mathrm{k}_{\mathrm{c}}\right)$, b adjacent Mn1 and Mn2 magnetic spins $\left(\theta_{\mathrm{Mn1}-\mathrm{Mn2}}\right)$, and $\mathbf{c}$ Mn magnetic spins and the $b$-axis $(\gamma)$. The solid and dashed lines correspond to cycloidal and helical structures, respectively. Error bars are also shown in the plots; some error bars are smaller than the data symbols. d Mn cycloidal magnetic structures of cell $a \times b \times c$ for the $x=0.020$ sample viewed from the $a$-axis at 3, 70, and $160 \mathrm{~K}$. Mn magnetic helices of cell $a \times b \times 3 c$ for the $x=0.020$ sample viewed from the $c$-axis at 190, 250, and $300 \mathrm{~K}$. The angles between different Mn spins are defined and given.

$\theta_{\mathrm{Mn} 1-\mathrm{Mn} 3}$ is further reduced (i.e., $23^{\circ}$ at $300 \mathrm{~K}$ for the $x=$ 0.020 sample). Moreover, the phase analysis, as shown in Fig. 6b, indicates that the angle between the nearest atoms of two Mn cycloidal or helical spirals $\left(\theta_{\mathrm{Mn1}-\mathrm{Mn} 2}\right)$ decreases (i.e., $19^{\circ}$ at $300 \mathrm{~K}$ for the $x=0.020$ sample). Consequently, the spins of all $\mathrm{Mn}$ atoms tend to continuously rotate toward the parallel arrangement of the $b$-axis, as shown in Fig. 6c, d; thus, an FM-like spin configuration is expected in Ni-containing samples.

In addition, Lorentz TEM is widely employed to investigate the real-space imaging of spiral magnetic structures $^{31,32}$. We imaged the magnetic domain structures in the $x=0.020$ sample using Lorentz TEM under a zero magnetic field between $140 \mathrm{~K}$ and $300 \mathrm{~K}$, as shown in Fig. 7 (details are shown in Supplementary Fig. S9). The studied thin specimen is near the [1-10] zone axis orientation confirmed by the SAED in the inset. At $293 \mathrm{~K}$, uniform and nanosized fine magnetic patterns with bright and dark contrast are repeatedly arranged perpendicular to the $c$-axis. Based on the over- and underfocused
Lorentz TEM images (Supplementary Fig. S9), a TIE was used to characterize the spin textures of the magnetic patterns. The yellow and blue straight stripe pairs reflect the regions with opposite in-plane magnetic inductions, as indicated by the color wheel. Together with the line profile of the alternative contrast intensity, the nearly sinusoidal changes in the inductions indicate that the spin order is probably spiral or fan-like. Note that only the inplane component of moments is presented by the Lorentz TEM. Therefore, the real 3D magnetic structures of this thin $\mathrm{MnCoSi}$ specimen should be resolved systematically by in situ Lorentz TEM in the future. The fine stripe-type magnetic domain can be observed in the studied temperature ranges of $140-293 \mathrm{~K}$. With increasing temperature, the width of the stripe increases. In addition, the spiral magnetic structure, which contains a higher harmonic modulation of magnetic order, clearly provides a pair of diffraction spots along the $c$-axis close to the (000) diffraction spots of SAED, as shown in the inset of Fig. 7b. Through quantitative analysis of these satellite spots, the 
(a)

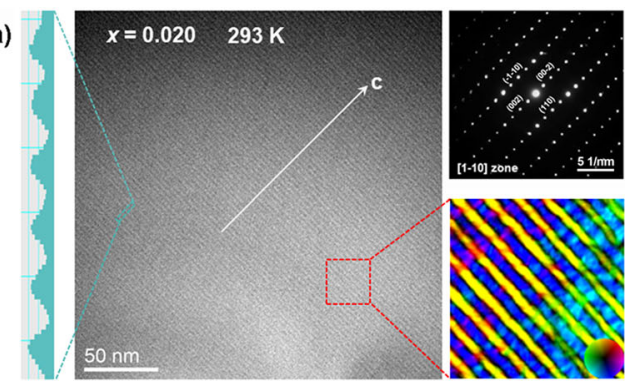

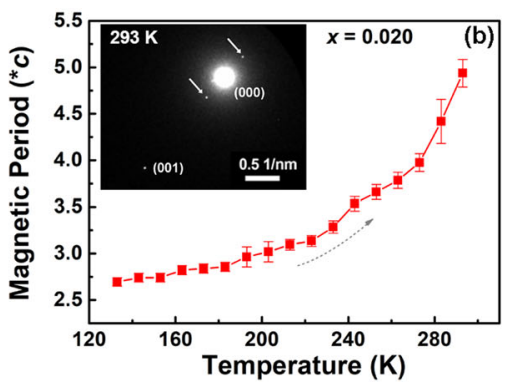

Fig. 7 Analysis of Lorentz TEM images. a Overfocused Lorentz TEM images of the domain structures at $293 \mathrm{~K}$ of an $x=0.020$ sample thin plate in zero magnetic field. The SAED pattern in the upper right indicates the [1-10] orientation. A line profile of the contrast intensity integrated in a dashed cyan region is shown on the left. The spin texture in the red dashed box obtained by TIE analysis of the Lorentz TEM data is shown in the lower right. b The period of the spiral magnetic structure obtained from SAED. SAED performed at a camera length of $7.8 \mathrm{~m}$ is shown in the inset.
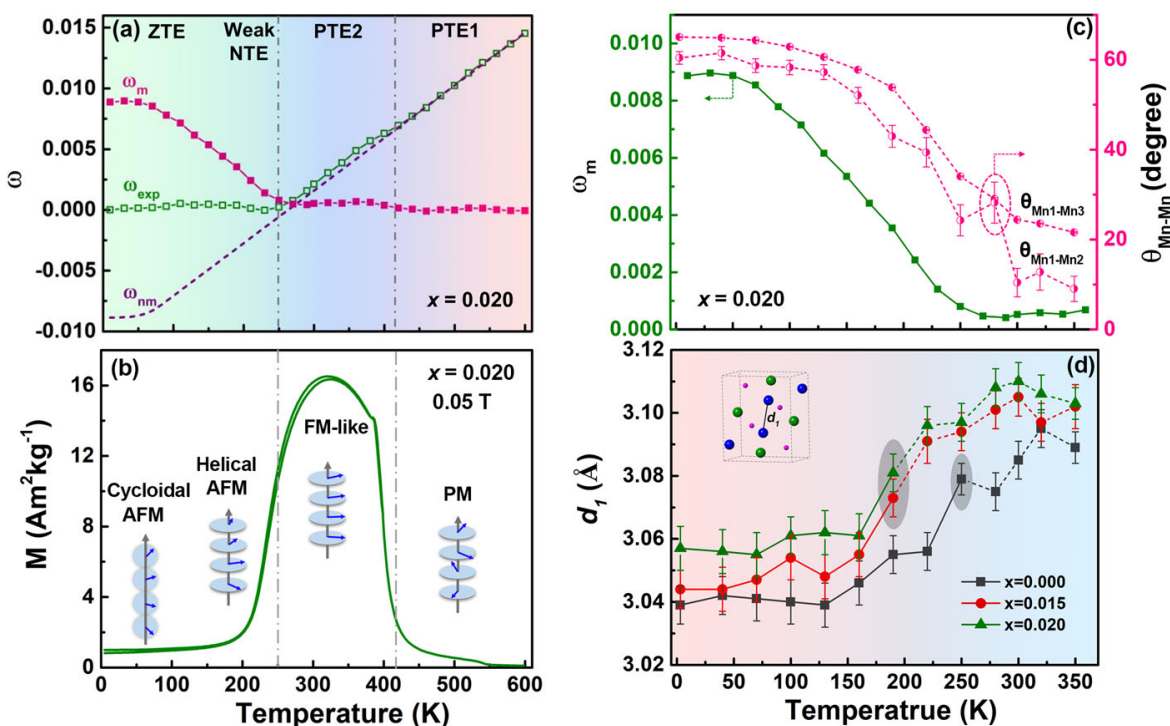

Fig. 8 Thermal expansion properties and evolution of the magnetic structure parameters. a Thermal expansion of sample $x=0.020$. The spontaneous volume magnetostriction $\left(\omega_{\mathrm{m}}\right)$ is calculated by subtracting the contribution of the nonmagnetic part $\left(\omega_{\mathrm{nm}}\right)$ from the experimental thermal expansion data $\left(\omega_{\text {exp }}\right)$. b M-T curves of sample $x=0.020$ at 0.05 T. $\mathbf{c}$ Temperature dependence of $\omega_{m}$ and adjacent angles between Mn spins. d Temperature dependence of the Mn-Mn distance $d_{1}$ obtained from Rietveld refinement of NPD patterns. $d_{1}$ is indicated in the inset, and the magnetic structure variation temperature is highlighted.

magnetic spiral period can be calculated, as shown in Fig. 7b. As the temperature increases, the period gradually increases and accelerates at $\sim 220 \mathrm{~K}$, which is consistent with the NPD data and further validates the coherent rotation of the magnetic spins.

\section{Discussion}

Near-ZTE behavior has been mentioned in terms of or observed in MnCoSi-based alloys ${ }^{20,33}$, while the origin of the effect was not discussed. The conventional mechanism underlying ZTE or NTE in magnetic alloys, such as La $(\mathrm{Fe}, \mathrm{Si})_{13}$ alloys and $\mathrm{RFe}_{2}$-based compounds ${ }^{11,14-16,34,35}$, originates from either a magnetic disorder to order transition or a large change in the magnetic moment.
Here, to quantitatively uncover the contribution of magnetism to the thermal expansion behavior of the $\mathrm{Mn}_{1-x} \mathrm{Ni}_{x} \mathrm{CoSi}$ system, the spontaneous volume magnetostriction $\omega_{\mathrm{m}}$ of sample $x=0.020$ is calculated by $\omega_{\mathrm{m}}=$ $\omega_{\exp }-\omega_{\text {nm }}$, in which $\omega_{\exp }$ is obtained from the experimental XRD results and $\omega_{n m}$ is fitted from the nonmagnetic phase based on the Debye-Grüneisen model $^{36,37}$, as shown in Fig. 8a. Combined with the M-T curve (Fig. 8b), the sample displays linear PTE behavior in the PM region. When the sample starts to enter the ordered FM-like state at below $410 \mathrm{~K}$, the experimental $\omega_{\exp }$ slightly deviates from the fitted $\omega_{\mathrm{nm}}$ due to the MVE. With a further decrease in the temperature, a smooth magnetic transition from the FM-like state to the spiral 
AFM state is observed, during which the thermal expansion behavior is significantly affected. Specifically, the negative role of magnetic $\omega_{\mathrm{m}}$ gently exceeds or completely counteracts the contribution from the lattice variation, which results in a weak NTE and a wide-temperature ZTE. Moreover, $\omega_{\mathrm{m}}$ and the angles between Mn spins exhibit similar temperature-dependent behavior (Fig. 8c), which indicates an intimate relationship between the anomalous thermal expansion and spin rotation of the helical magnetic structure in MnCoSi-based alloys.

Notably, thermally induced coherent spin rotation is also observed in stoichiometric $\mathrm{MnCoSi}$. During heating, the angles $\theta_{\mathrm{Mn} 1-\mathrm{Mn} 3}$ and $\theta_{\mathrm{Mn} 1-\mathrm{Mn} 2}$ decrease from $70^{\circ}$ and $61^{\circ}$ to $38^{\circ}$ and $32^{\circ}$, respectively. Then, the rotation is forced to cease by the disordered PM state. Therefore, this weak and partial spin rotation brings about only a small fluctuation in the thermal expansion behavior of stoichiometric MnCoSi (shown in Fig. 2d), for which a PTE is observed over the entire temperature range. The unusual magnetic tricritical behavior of $\mathrm{MnCoSi}$ results in flexible tunability of the magnetic state ${ }^{20}$. It is widely reported that the magnetic state of this Mn-based orthorhombic alloy with space group Pnma is extremely sensitive to the Mn-Mn distance $d_{1}{ }^{19,38}$. In this work, the introduction of $\mathrm{Ni}$ atoms can produce "chemical pressure" on the crystal lattice and change $d_{1}$. As shown in Fig. $8 \mathrm{~d}, d_{1}$ increases with increasing $\mathrm{Ni}$ content; correspondingly, nonlinear AFM tends to be FM. Therefore, the enhanced FM interaction leads to an obvious transition from the cycloidal or helical AFM state to an FM-like state, during which the magnetic spins further coherently rotate to the $b$-axis. Due to robust magnetoelastic coupling ${ }^{20,33}$, this strong rotation gives rise to giant spontaneous magnetostriction, particularly the sharp contraction of the $a$-axis, and leads to the emergence of anomalous NTE or ZTE behavior in the homogeneous phase. In addition, our results suggest that an appropriate internal or external stimulus, such as doping with elements, introducing vacancies or applying hydrostatic pressure or a magnetic field, can strengthen the FM interaction and establish this spiral AFM-FM-type transition, inducing ZTE in MnCoSi-based alloys.

Note that the change in magnetic structure from cycloidal to helical spiral cannot be clearly revealed in the evolution of lattice parameters and magnetic properties of polycrystalline samples. Due to the intimate relations between the magnetic state and $\mathrm{Mn}-\mathrm{Mn}$ separation $^{20}$, an evident step change of $d_{1}$ (Fig. 8d) can be observed that corresponds to the change in the spiral AFM structure in the MnCoSi-based alloy. In addition, owing to the distinct easy magnetization planes of cycloidal ( $b c$ plane) and helical ( $a b$ plane) spirals, the magnetic structures can be effectively distinguished by magnetic characterization of MnCoSi single crystals.

\section{Conclusions}

In summary, a wide-temperature ZTE effect and a new cycloidal spiral AFM structure were found in orthorhombic $\mathrm{Mn}_{1-x} \mathrm{Ni}_{x} \mathrm{CoSi}$ alloys. Systematic magnetic measurements and in situ XRD, NPD, and Lorentz TEM characterization indicated that the introduction of $\mathrm{Ni}$ can enhance the FM interaction and induce a transition from the spiral AFM state to an FM-like state. During this transition, the spin lying in the $b c$ or $a b$ plane rotates uniformly, leading to drastic changes in the lattice parameters due to magnetoelastic coupling, which results in ZTE behavior. Moreover, this new mechanism sheds light on magnetic materials that possess this spiral AFM-FMtype transition, and ZTE or NTE materials may be discovered or designed.

\section{Acknowledgements}

This work was supported by the National Key R\&D Program of China (2017YFA0206303), the National Natural Science Foundation of China (No. 11974406), the Strategic Priority Research Program (B) of the Chinese Academy of Sciences (CAS) (XDB33000000), and the China Postdoctoral Science Foundation (No. 2020M680735). Z.X. Cheng thanks the Australian Research Council for financial support (DP190100150).

\section{Author details \\ ${ }^{1}$ Beijing National Laboratory for Condensed Matter Physics, Institute of Physics, Chinese Academy of Sciences, 100190 Beijing, China. ${ }^{2}$ Institute for \\ Superconducting and Electronic Materials, Innovation Campus, University of Wollongong, Squires Way, North Wollongong, NSW 2500, Australia. ${ }^{3}$ Neutron Group, National Synchrotron Radiation Research Center, Hsinchu 30076, \\ Taiwan. ${ }^{4}$ Songshan Lake Materials Laboratory, 523808 Dongguan, Guangdong, China}

\section{Author contributions}

J.L. and W.W. proposed the idea and designed the experiments. J.L. and B.D. prepared the samples. B.D. and Y.Y. performed TEM and analyzed the related results. J.L. and X.X. measured the magnetic properties. Z.C., J.W., and C.W. performed NPD. J.L., J.W., and G.W. analyzed the NPD results. All authors discussed the results. J.L. and W.W. wrote and developed the manuscript.

Conflict of interest

The authors declare no competing interests.

\section{Publisher's note}

Springer Nature remains neutral with regard to jurisdictional claims in published maps and institutional affiliations.

Supplementary information The online version contains supplementary material available at https://doi.org/10.1038/s41427-021-00338-4.

Received: 7 July 2021 Revised: 13 September 2021 Accepted: 23 September 2021.

Published online: 5 November 2021

\section{References}

1. Chen, J., Hu, L., Deng, J. X. \& Xing, X. R. Negative thermal expansion in functional materials controllable thermal expansion by chemical modifications. Chem. Soc. Rev. 44, 3522-3567 (2015).

2. Mary, T. A., Evans, J. S. O., Vogt, T. \& Sleight, A. W. Negative thermal expansion from 0.3 to 1050 Kelvin in $\mathrm{ZrW}_{2} \mathrm{O}_{8}$. Science 272, 90-92 (1996).

3. Hu, P., Chen, J., Deng, J. \& Xing, X. Thermal expansion, ferroelectric and magnetic properties in $(1-x) \mathrm{PbTiO}_{3}-x \mathrm{Bi}\left(\mathrm{Ni}_{1 / 2} \mathrm{Ti}_{1 / 2}\right) \mathrm{O}_{3}$. J. Am. Chem. Soc. 132, 1925-1928 (2010). 
4. Azuma, M. et al. Colossal negative thermal expansion in $\mathrm{BiNiO}_{3}$ induced by intermetallic charge transfer. Nat. Commun. 2, 347 (2011).

5. Yilmaz, S. \& Dunand, D. C. Finite-element analysis of thermal expansion and thermal mismatch stresses in a $\mathrm{Cu}-60 \mathrm{vol} \% \mathrm{ZrW}_{2} \mathrm{O}_{8}$ composite. Compos. Sci. Technol. 64, 1895-1898 (2004).

6. Miracle, D. B. Metal matrix composites-From science to technological significance. Compos. Sci. Technol. 65, 2526-2540 (2005).

7. Liu, J. et al. Realization of zero thermal expansion in $\mathrm{La}(\mathrm{Fe}, \mathrm{Si})_{13}$-based system with high mechanical stability. Mater. Des. 148, 71-77 (2018).

8. Guillaume, C. E. - Recherches sur les aciers au nickel. Dilatations aux temperatures elevees; resistance electrique. Cr. Acad. Sci. 125, 18 (1897).

9. Wada, H. \& Shiga, M. Thermal expansion anomaly and Invar effect of $\mathrm{Mn}_{1}$. ${ }_{x}$ Co B. J. Mag. Mag. Mater. 104, 1925-1926 (1992).

10. Salvador, J. R., Guo, F., Hogan, T. \& Kanatzidis, M. G. Zero thermal expansion in $\mathrm{YbGaGe}$ due to an electronic valence transition. Nature 425, 702-705 (2004).

11. Wang, W. et al. Zero thermal expansion in $\mathrm{NaZn} \mathrm{n}_{13}$-type $\mathrm{La}(\mathrm{Fe}, \mathrm{Si})_{13}$ compounds. Phys. Chem. Chem. Phys. 17, 2352-2356 (2015).

12. Dan, S., Mukherjee, S., Mazumdar, C. \& Ranganathan, R. Zero thermal expansion with high Curie temperature in $\mathrm{Ho}_{2} \mathrm{Fe}_{16} \mathrm{Cr}$ alloy. RSC Adv. 6, 94809-94814 (2016).

13. Li, W. et al. Strong coupling of magnetism and lattice induces near-zero thermal expansion over broad temperature windows in $\mathrm{ErFe}_{10} \mathrm{~V}_{2-x} \mathrm{Mo}_{x} \mathrm{Com}$ pounds. CCS Chem. 2, 1009-1015 (2020).

14. Song, $\mathrm{Y}$. et al. Zero thermal expansion in magnetic and metallic $\mathrm{Tb}(\mathrm{Co}, \mathrm{Fe})_{2}$ intermetallic compounds. J. Am. Chem. Soc. 140, 602-605 (2018).

15. Song, $Y$. et al. Transforming thermal expansion from positive to negative: the case of cubic magnetic compounds of $(\mathrm{Zr}, \mathrm{Nb}) \mathrm{Fe}_{2}$. J. Phys. Chem. Lett. 11 1954-1961 (2020).

16. Song, $\mathrm{Y}$. et al. Negative thermal expansion in $(\mathrm{Sc}, \mathrm{Ti}) \mathrm{Fe}_{2}$ induced by an unconventional magnetovolume effect. Mater. Horiz. 7, 275-281 (2020).

17. Takenaka, K. \& Takagi, H. Giant negative thermal expansion in Ge-doped antiperovskite manganese nitrides. Appl. Phys. Lett. 87, 261902 (2005).

18. Miao, P. et al. Large magnetovolume effect induced by embedding ferromagnetic clusters into antiferromagnetic matrix of cobaltite perovskite. Adv. Mater. 1605991, 1-6 (2017).

19. Gercsi, Z. \& Sandeman, K. G. Structurally driven metamagnetism in MnP and related Pnma compounds. Phys. Rev. B 81, 224426 (2010).

20. Barcza, A., Gercsi, Z., Knight, K. S. \& Sandeman, K. G. Giant magnetoelastic coupling in a metallic helical metamagnet. Phys. Rev. Lett. 104, 247202 (2010).
21. Sandeman, K. G. et al. Negative magnetocaloric effect from highly sensitive metamagnetism in $\mathrm{CoMnSi}_{1-x} \mathrm{Ge}_{x}$. Phys. Rev. B 74, 224436 (2006).

22. Gong, Y. Y. et al. Textured, dense and giant magnetostrictive alloy from fissile polycrystal. Acta Mater. 98, 113-118 (2015).

23. Liu, J. et al. Large, low-field and reversible magnetostrictive effect in MnCoSibased metamagnet at room temperature. J. Mater. Sci. Technol. 76, 104-110 (2021).

24. Morrison, $\mathrm{K}$. et al. The magnetocaloric performance in pure and mixed magnetic phase CoMnSi. J. Phys. D: Appl. Phys. 43, 195001 (2010).

25. Rietveld, H. M. A profile refinement method for nuclear and magnetic structures. J. Appl. Crystallogr. 2, 65-71 (1969).

26. Rodriguez-Carvajal, J. Recent advances in magnetic structure determination by neutron powder diffraction. Phys. B 192, 55-69 (1993).

27. Nizioł, S., Bombik, A., Bażela, W., Szytuła, A. \& Fruchart, D. Mgnetic phase diagram of $\mathrm{Co}_{x} \mathrm{Ni}_{1-x} \mathrm{MnGe}$. Solid State Commun. 42, 79-83 (1982).

28. Morrison, K. et al. Measurement of the magnetocaloric properties of $\mathrm{CoMn}_{0.95} \mathrm{Fe}_{0.05} \mathrm{Si}$ : Large change with Fe substitution. Phys. Rev. B 78, 134418 (2008).

29. Izyumov, Y. A., Naish, V. E. \& Ozerov, R. P. Neutron Diffraction of Magnetic Materials (Consultants Bureau, Plenum Publishing Corporation, 1991).

30. Baumfeld, O. L. Magnetoelastic Coupling and Tricritical Metamagnetism. PhD dissertation, Imperial College London (2017).

31. Uchida, M., Onose, Y., Matsui, Y. \& Tokura, Y. Real-space observation of helical spin order. Science 311, 359-361 (2006).

32. Togawa, Y. et al. Chiral magnetic soliton lattice on a chiral helimagnet. Phys. Rev. Lett. 108, 107202 (2012).

33. Barcza, A. et al. Magnetoelastic coupling and competing entropy changes in substituted CoMnSi metamagnets. Phys. Rev. B 87, 064410 (2013).

34. Li, $\mathrm{S}$. et al. Zero thermal expansion achieved by an electrolytic hydriding method in $\mathrm{La}(\mathrm{Fe}, \mathrm{Si})_{13}$ compounds. Adv. Funct. Mater. 1604195, 1 (2017).

35. Huang, R. et al. Giant negative thermal expansion in $\mathrm{NaZn}_{13}$-type $\mathrm{La}(\mathrm{Fe}, \mathrm{Si}, \mathrm{Co})_{13}$ compounds. J. Am. Chem. Soc. 135, 11469-11472 (2013).

36. Sayetat, F., Fertey, P. \& Kessler, M. An easy method for the determination of Debye temperature from thermal expansion analyses. J. Appl. Crystallogr. 31, 121-127 (1998).

37. Moriya, T. \& Usami, K. Magneto-volume effect and Invar phenomena in ferromagnetic metals. Solid State Commun. 34, 95-99 (1980).

38. Staunton, J. B., dos Santos Dias, M., Peace, J., Gercsi, Z. \& Sandeman, K. G. Tuning the metamagnetism of an antiferromagnetic metal. Phys. Rev. B 87 060404(R) (2013). 